\title{
Lesions of the central nervous system in leukemia: Pathological and magnetic resonance imaging features at presentation in 14 patients
}

\author{
JING LIU $^{1 *}$, YAMING WANG ${ }^{2 *}$, XUEFEI SUN ${ }^{1}$, SHUO LIU ${ }^{3}$, ZHENKUN YU $^{4}$, \\ NAN JI ${ }^{5}$, SHENGJUN SUN ${ }^{6}$ and YUANBO LIU ${ }^{1}$
}

\begin{abstract}
${ }^{1}$ Department of Hematology, Beijing Tiantan Hospital, Capital Medical University, Beijing 100050; ${ }^{2}$ Department of Neurosurgery, Navy General Hospital, Beijing 100048, P.R. China; ${ }^{3}$ Duke Clinical Research Institute, Duke University, Durham, NC 27705, USA; ${ }^{4}$ Department of Otorhinolaryngology, Head and Neck Surgery, Nanjing Tongren Hospital, Southeast University, Nanjing 211100; ${ }^{5}$ Department of Neurosurgery; ${ }^{6}$ Neuroimaging Center, Beijing Tiantan Hospital, Capital Medical University, Beijing 100050, P.R. China
\end{abstract}

Received November 7, 2015; Accepted January 26, 2017

DOI: $10.3892 / \mathrm{ol} .2017 .7192$

\begin{abstract}
The present study aimed to characterize the specific pathology and magnetic resonance imaging (MRI) findings observed in patients with leukemia with central nervous system (CNS) lesions, and to determine their value in the management of such patients. Lesions of the CNS were observed during and following treatment of leukemia. The data from stereotactic biopsy-proven pathology (12 patients) and MRI examinations (14 patients) were retrospectively evaluated. Proton-magnetic resonance-spectroscopy was performed in three patients. Factors that predisposed to lesions of the CNS were reviewed from the patient medical records. Among the 14 patients, eight had CNS leukemia, four had a CNS infection and two had a neurodegenerative disorder (one leukoencephalopathy and one glial cell hyperplasia). The clinical diagnosis based on clinical symptoms, signs and MRI features was not consistent with the pathological diagnosis in two patients. In one patient, the clinical diagnosis was a CNS infection; however, the patient's pathological diagnosis was CNS leukemia. In the other patient, the clinical diagnosis was CNS leukemia, but the pathological diagnosis was glial cell hyperplasia. CNS lesions in leukemia have a wide range of causes. Apart from the relapse of leukemia in the CNS, there are treatment-associated neurotoxicities and infections that are caused by immunocompromised states.
\end{abstract}

Correspondence to: Dr Yuanbo Liu, Department of Hematology, Beijing Tiantan Hospital, Capital Medical University, 6 Tiantan Xili, Dongcheng, Beijing 100050, P.R. China

E-mail: yuanbol@ccmu.edu.cn

*Contributed equally

Key words: leukemia, central nervous system lesions, stereotactic biopsy, magnetic resonance imaging, pathology
As numerous leukemia-associated CNS lesions are treatable, early diagnosis is essential.

\section{Introduction}

Leukemia is the most common form of hematological malignancy and it has an incidence rate of 3-4/10 million between 1988 and $2002(1,2)$ and 5.17/10 million in china between 2003 and 2007 (3). Due to the continuous optimization of treatment and supportive care, the survival rate for patients with leukemia has been prolonged significantly over the last few decades $(4,5)$. As a consequence of these improvements, the frequency of central nervous system (CNS) involvement in leukemia has increased $(6,7)$. The diagnosis of CNS leukemia should be considered in the differential diagnosis of patients with leukemia and CNS lesions, including those with CNS infection and neurodegenerative disorders following leukemia treatment. CNS lesions in leukemia may occur due to the disease itself, or the treatment (8-13). Disease-associated CNS complications may consist of leukemic cell involvement of the meninges, parenchyma, and cerebrovasculature (8), whilst treatment-associated CNS complications may include leukoencephalopathy, inflammatory demyelinating polyradiculoneuropathy, infections, vascular disorders and secondary tumors (9-12).

The clinical presentations of CNS lesions in leukemia vary. Diagnosing the nature of CNS lesions is often challenging, as there is a varied set of causes (14). There are no pathognomonic imaging features for CNS lesions in leukemia, and the histological verification of a brain biopsy remains the gold standard for diagnosis $(15,16)$. As numerous CNS lesions in leukemia are curable, early diagnosis is essential for their proper management (13). Several studies have reported the usefulness of magnetic resonance imaging (MRI) and computed tomography (CT) in the diagnosis of CNS lesions in leukemia $(13,17-20)$. However, there are a limited number of reports concerning the pathological features of CNS lesions in leukemia. 
The present study aimed to combine the MRI and pathological findings observed in 14 patients with leukemia with CNS lesions, characterize these features and determine their value in the diagnosis of such patients. Doing so may aid reaching the correct diagnosis in the future and potentially allow treatment to be conducted without delay.

\section{Patients and methods}

Patients. The clinical data, MRI features and pathology results of 14 patients (11 male and 3 female), whose ages ranged from 7 to 60 years old, were retrospectively reviewed at Beijing Tiantan Hospital (Capital Medical University, Beijing, China) between April 2003 and May 2015 (Table I). The patients had various types of leukemia, including 8 cases of acute lymphoblastic leukemia (ALL; 4 B-cell-ALL, 2 T-cell-ALL and 2 unknown type-ALL), 4 cases of acute myelogenous leukemia (AML; 2 M5, 1 M4 and 1 unknown type), 1 case of acute promyelogenous leukemia (APL) and 1 case of chronic myelomonocytic leukemia (CMML).

The majority of patients presented with nonspecific symptoms, including seizure, headache, nausea, vomiting and a change in mental status. Few patients had focal neurological deficiencies, including limb weakness and vision loss. Systemic symptoms, including fever, night sweat and weight loss, were common.

Patient medical records were reviewed with particular attention to the type of treatment given, time of onset of neurological symptoms, the interval between the onset of neurological symptoms and final treatment for leukemia, cerebrospinal fluid (CSF) results (pressure, Pandy test, quantitative protein levels, cell count and bacterial/fungal cultures), outcome of the CNS lesions, MRI findings in 14 patients, pathological features in 12 patients and the consistency of the clinical diagnosis and pathological diagnosis (Table I). The study protocol was approved by the Ethics Committee of Beijing Tiantan Hospital (Beijing, China). All of the patients provided written informed consent.

Imaging. Two experienced neuroradiologists (Beijing Tiantan Hospital, Capital Medical University, Beijing, China) retrospectively and independently evaluated the images. All scans were reviewed, noting the brain lesion locations, size, margin and signal characteristics, as well as the presence of perilesional edema, mass-effect, hemorrhage, necrosis and meningeal enhancement.

All of the patients were imaged using T1- and T2-weighted and post-contrast T1-weighted scans (0.1-0.15 mmol gadolinium-diethylenetriaminepenta-acetate $/ \mathrm{kg}$ body weight). Proton-magnetic resonance-spectroscopy $\left({ }^{1} \mathrm{H}-\mathrm{MRS}\right)$ was performed in 3 patients. MRI was performed on a $1.5-\mathrm{T}$ Siemens MAGNETOM ${ }^{\circledR}$ Avanto machine (Siemens AG, Munich, Germany). On this machine, T1 images were fast spin-echo sequences with repetition time (TR), $500 \mathrm{~ms}$ and echo time (TE), $7.8 \mathrm{~ms}$. T2 images were fast spin-echo sequences with TR, 3,630 ms and TE, $93 \mathrm{~ms}$. The pre- and post-contrast $\mathrm{T} 1$ sequences were obtained along the three orthogonal planes. The T2 sequences were obtained axially. The pre- and post-contrast T1 sequences were isotropic, ultrafast spoiled gradient echo sequences (TR, $8.3 \mathrm{~ms}$ and TE,
$3.8 \mathrm{~ms}$ ). The T2 sequences were 5-mm axial fast spin echo sequences with TR, 4,000 ms and TE, $110 \mathrm{~ms} .{ }^{1} \mathrm{H}-\mathrm{MRS}$ was obtained with a long echo time (135 ms) as a multivoxel 2D exam encompassing the lesion and normal white matter.

Stereotactic biopsy. Among the 14 patients with leukemia, 12 were pathological, as confirmed by stereotactic biopsy with a framework of the stereotactic surgery planning system (AeroTech) and robot-assisted planning of frameless stereotactic surgery (Computer Stereotactic Assistant, type R; CSA-R type), in order to place the stereotactic frame on the head or post four marker points on the head and then used MRI to locate the lesion. Through the local area network, images were uploaded into the workstation to formulate the surgical plan, determine the biopsy site, avoid the important functional areas and select the appropriate cranial puncture point and best surgical puncture path (Figs. 1A and 2A). A biopsy needle was inserted following a hole being drilled with a 3-mm diameter drill. Four or five pieces of tissues measuring $1.0 \times 0.3 \times 0.3 \mathrm{~cm}$ were removed from the lesion area. An intraoperative snap-frozen tissue section used liquid nitrogen and a conventional paraffin-embedded tissue section was sent for evaluation (Figs. 3A and 4A). Following the biopsy, the equipment was withdrawn and the wound was sutured.

\section{Results}

Among the 14 patients, the causes of CNS lesions were divided into three groups. The first group had CNS leukemia $(n=8$; Fig. 1B), the second group had CNS infection $(n=4$; Fig. 3B and C) and the third group had a neurodegenerative disorder ( $n=2,1$ leukoencephalopathy and 1 glial cell hyperplasia 1; Fig. 2B and C). CNS leukemia included 1 APL case, 3 ALL (B cell) cases, 2 ALL ( $\mathrm{T}$ cell) cases, 1 CMML case and 1 AML (M5b) case. CNS infection included 3 AML cases and 1 ALL case (T cell), whereas neurodegenerative disorders included 2 ALL cases (B cell).

All the patients received chemotherapy, and 6 received hematopoietic stem cell allotransplantation (allo-HSCT). In group 1, CNS leukemia occurred prior to chemotherapy $(n=1$, case 8 ), during chemotherapy ( $\mathrm{n}=1$, case 2$)$, following chemotherapy patients obtained complete remission $(n=3$, case 1,5 , 6 and case 5 with systemic relapse) and following allo-HSCT ( $n=3$, case $3,4,7$ and case 4 with systemic relapse).

In the second group, 2 patients with CNS infection following allo-HSCT with graft vs. host disease (GVHD; 1 GVHD of the intestinal tract and 1 GVHD of the liver; cases 9 and 10, respectively) were given immunosuppressive therapy, including cyclosporin and/or antithymocyte globulin against GVHD. Another 2 patients had CNS infection during the second and fourth courses of chemotherapy for anti-leukemic treatment (cases 11 and 12, respectively).

In group 3, 1 patient had leukoencephalopathy following 3 courses of systemic chemotherapy and 5 intrathecal injections (case 13). Another patient had glial cell hyperplasia 1 month following high-dose chemotherapy and allo-HSCT (case 14).

The MRI features in patients with CNS leukemia indicated multiple, scattered, round solid nodules of lesions in the brain parenchyma, a slightly long or equal T1 and 


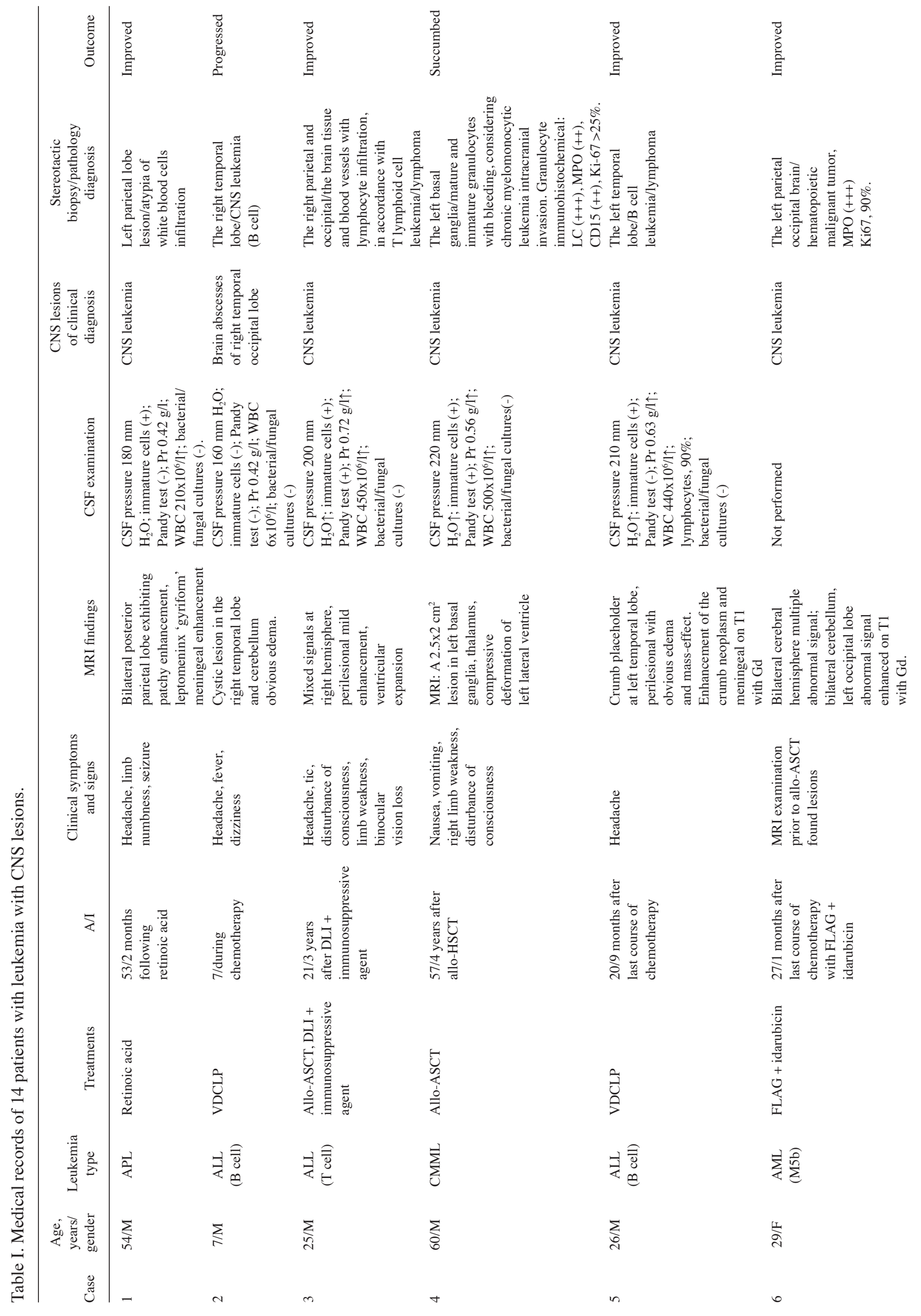




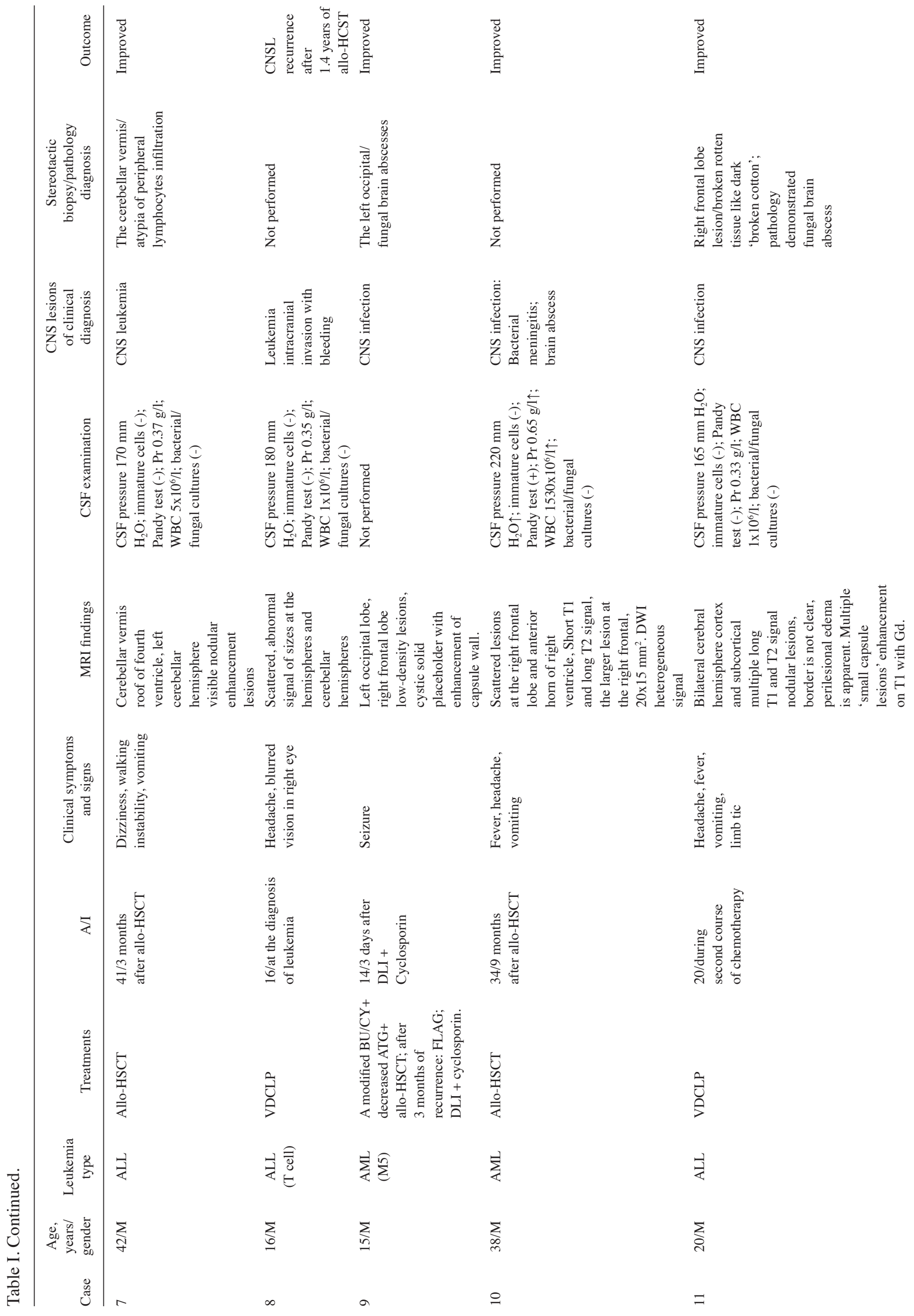




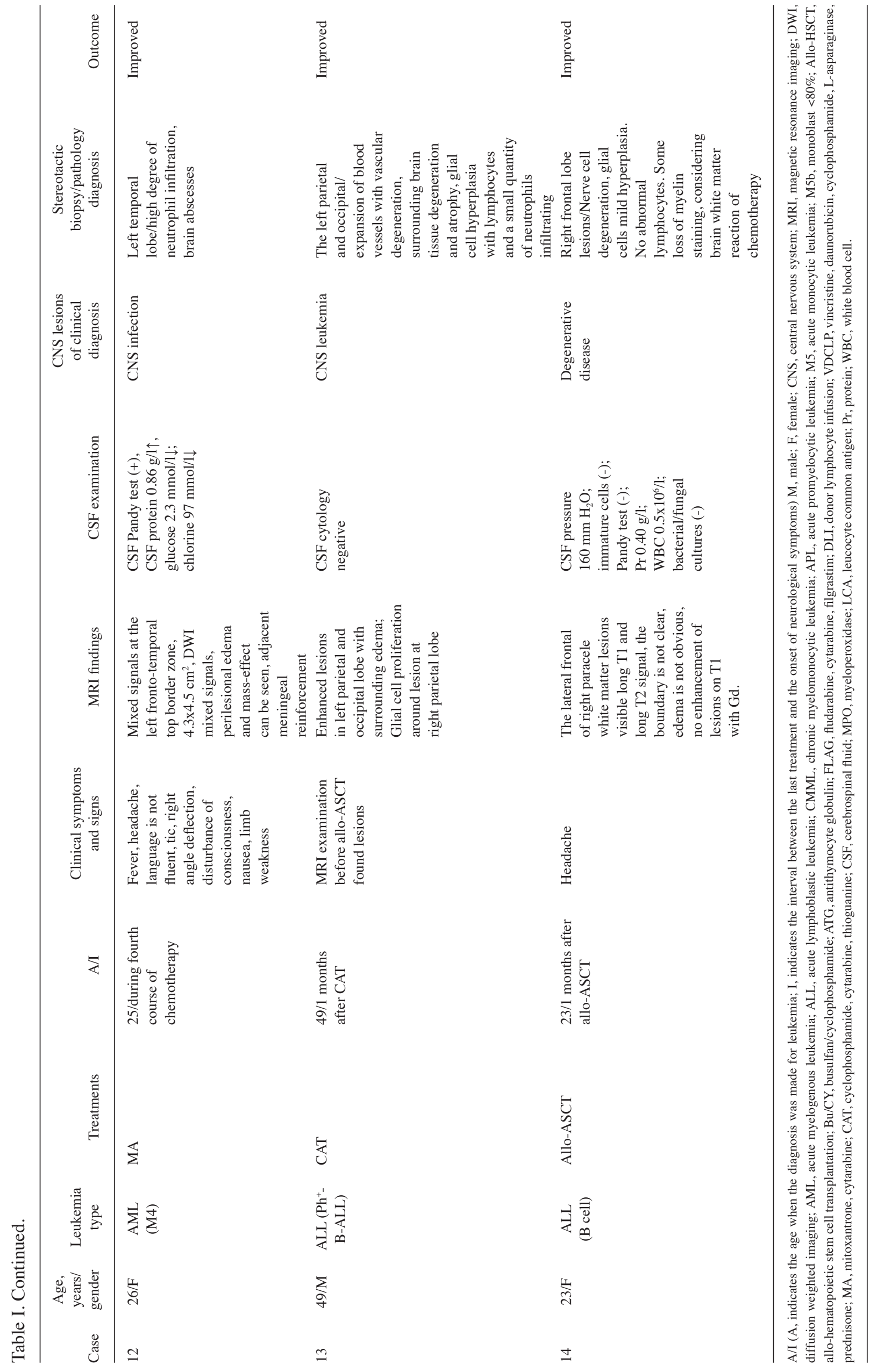


A

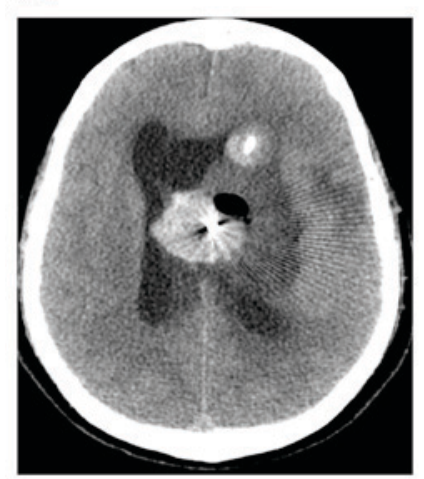

C

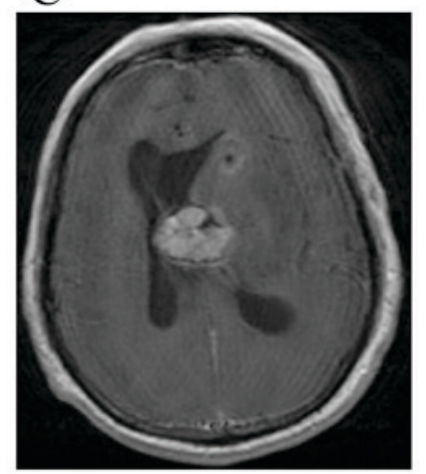

B

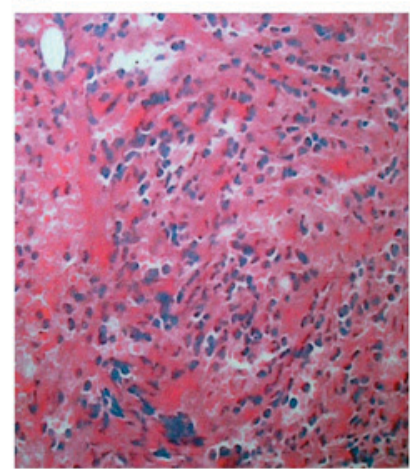

$\mathrm{D}$

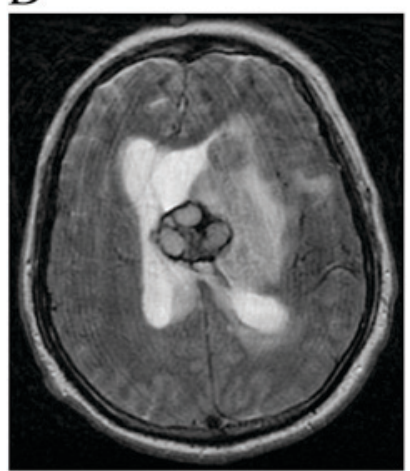

Figure 1. The MRI, biopsy site and pathology of a 60-year-old male with chronic myelomonocytic leukemia. (A) The robot-assisted planning of frameless stereotactic surgery reveals the biopsy site; seed artifacts are visible. (B) Pathology reveals mature and immature granulocytes with bleeding and this was considered to be chronic myelomonocytic leukemia intracranial invasion. (C) Following intravenous contrast injection, MRI revealed a $2.5 \times 2 \mathrm{~cm}$ intense enhancement lesion in the left basal ganglia, at the thalamus, a lump to the left ventricle, involvement of the corpus callosum and compressive deformation of the left lateral ventricle. (D) T2-weighted MRI showed mixed signal lesion with perilesional edema. MRI, magnetic resonance imaging.

long T2 signal and apparent perilesional edema as well as an enhanced mass effect (Fig. 1C and D). ${ }^{1} \mathrm{H}-\mathrm{MRS}$ was performed in 3 patients with CNS leukemia (case 1,2 and 3). ${ }^{1} \mathrm{H}-\mathrm{MRS}$ revealed a marked increase in choline (Cho), and a marked reduction in $\mathrm{N}$-acetylaspartate (NAA) and creatinine $(\mathrm{Cr})$ peaks in the center of the lesion. However, in perilesional brain tissue, a slight decrease in NAA and no increase in Cho peaks were observed. MRI findings in patients with CNS infection exhibited scattered, small capsule lesions, a short/long T1 and long T2 signal, an unclear border and apparent perilesional edema (Figs. 3D-F, 4B-D). MRI findings in patients with neurodegenerative disorders revealed glial cell proliferation around the lesion and white matter lesions that were visible on a long $\mathrm{T} 1$ and long T2 signal, whilst edema was not apparent (Fig. 2D).

Except for cases 8 and 10,12 patients obtained a pathological diagnosis through stereotactic biopsy. The clinical diagnosis was not consistent with the pathological diagnosis in 2 patients (cases 2 and 13). One patient's clinical diagnosis was CNS infection, although the pathological diagnosis was CNS leukemia (case 2). Another patient's clinical diagnosis and pathological diagnosis was CNS leukemia and glial cell hyperplasia, respectively (case 13). Among the 14 patients with

A

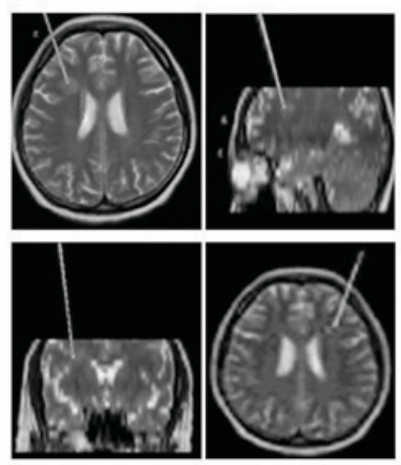

$\mathrm{C}$

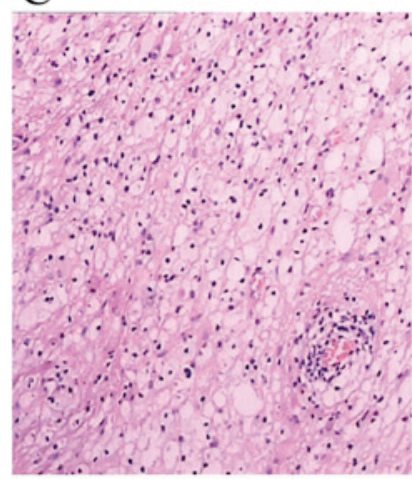

B

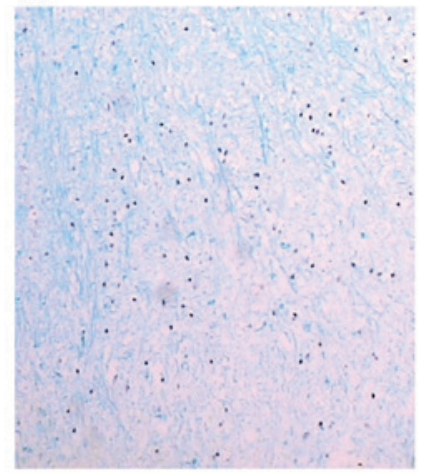

$\mathrm{D}$

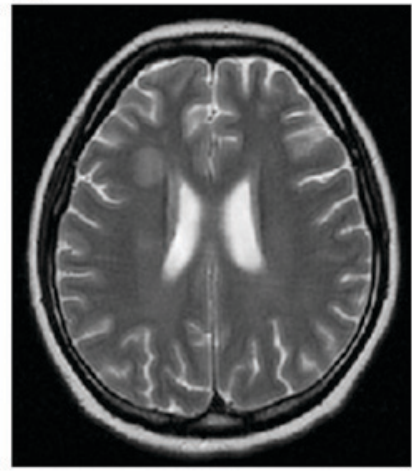

Figure 2. The MRI, biopsy site and pathology of a 23-year-old female with acute lymphoblastic leukemia. (A) The lesion of the right frontal lobe white matter was subjected to stereotactic biopsy. (B) Glial cell hyperplasia; a large number of foam cells and inflammatory cells infiltrated the perivascular region. (C) Immunohistochemical staining showing demyelination, axon fracture and degeneration. Cells are positive for glial fibrillary acidic protein. (D) T2-weighted MRI revealed a long T2 signal lesion on the lateral frontal lobe of the right paracele,, with an unclear boundary, non-evident edema. MRI, magnetic resonance imaging.

leukemia, the outcomes of 12 patients improved, 1 disease progression (case 2) and 1 succumbed (case 4).

\section{Discussion}

Leukemia with CNS involvement is not uncommon in clinical practice (13). In the present study, 14 patients with leukemia and CNS lesions were evaluated. It was observed that MRI aided the characterization of CNS lesions caused by the leukemic involvement of CNS structures, treatment-associated CNS complications and CNS infections due to immunocompromised states. However, two clinical diagnoses were not consistent with the pathological diagnoses, and pathological verification of brain biopsy tissues remains the gold standard for diagnosis (21-23).

CNS leukemia is more common in childhood, with hyperleukocytic acute leukemia, ALL and M5 often occurring following complete remission (4,6,7). Over the last two decades, clinical trials have significantly improved the response rates in patients with leukemia. Adults with ALL have a 60-90\% chance of reaching a first complete remission following combination chemotherapy (24-26). Advancements in the understanding of disease biology, adaptations to anti-leukemic treatment and better supportive care have all contributed to 
A

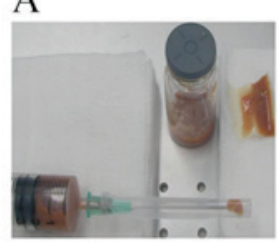

D

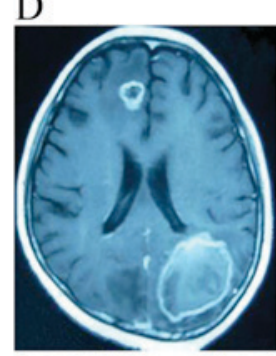

B

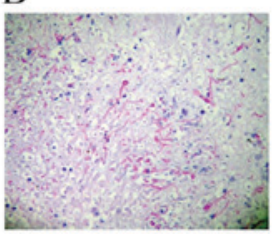

E

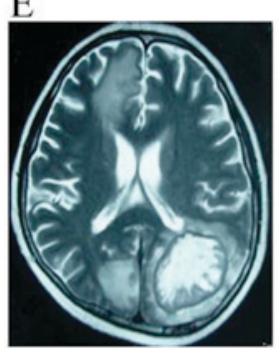

C

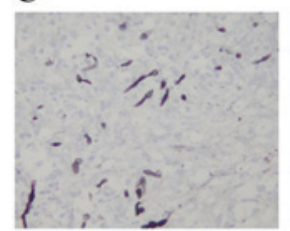

$\mathrm{F}$

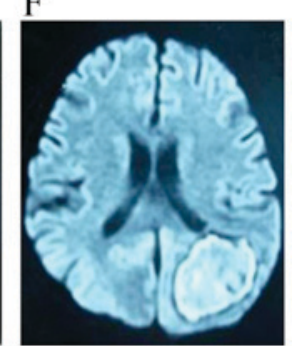

Figure 3. The MRI, biopsy material and pathology of a 15-year-old male with acute myelogenous leukemia (M5, acute monocytic leukemia). (A) Stereotactic biopsy was performed to obtain $4.5 \mathrm{ml}$ cystic fluid from the left occipital lobe. (B) Nerve cells degenerated in the lesion, gitter cells and reactive astrocyte hyperplasia were visible. (C) Blue-stained myelin debris may be observed in gitter cells. (D) T1-weighted MRI with gadopentetic acid revealed the left occipital lobe and right frontal lobe low-density lesions, and cystic solid placeholder with enhancement of the capsule wall. (E) T2-weighted MRI shows long T2 signal lesions with perilesional edema. (F) Diffusion-weighted imaging exhibits a high signal within the lesion. MRI, magnetic resonance imaging.

these improvements $(6,7)$. However, CNS involvement is still a primary cause of mortality and has become a major limitation to long-term survival (27). CNS-directed treatment is a significant contributing factor in improving the survival rate of patients with ALL. This essential treatment decreases the rate of CNS relapse, in addition to reducing the incidence of bone marrow recurrence. While $<5 \%$ of patients with ALL present with overt CNS leukemia, $>50 \%$ will develop CNS disease in the absence of prophylactic CNS-directed treatment (28). In the present study, 8 patients had CNS leukemia due to the disease itself. Cases 3, 4 and 7 developed CNS leukemia following allo-HSCT. CNS relapse subsequent to allo-HSCT indicates a poor prognosis in patients with leukemia $(29,30)$. Preventing CNS relapse following allo-HSCT remains a therapeutic challenge, and the criteria for post-HSCT CNS prophylaxis have yet to be addressed (27).

As chemotherapy drugs for the treatment of leukemia (anthracyclines, vinca alkaloids, cyclophosphamide) exhibit poor penetration of the blood brain barrier (BBB), the CNS becomes a 'shelter' for leukemia cells. CNS involvement is associated with a poor prognosis. However, methotrexate and cytarabine display a moderate capability to cross the BBB at high doses sufficient to obtain therapeutic concentrations within the CNS. However, as well as CNS radiation therapy (31), this contributes to neurotoxicities. Examples of neurotoxicity include leukoencephalopathy and spontaneous intracranial hemorrhage $(11,32-34)$. Infection may be caused by leukemia itself, as well as the bone marrow suppression observed with intense chemotherapy (13). In the present study, two patients developed a treatment-associated neurodegenerative disorder due to systemic high-dose chemotherapy and/or intrathecal injections. In a total of 4 patients, CNS infections occurred in the bone marrow suppression period during

A

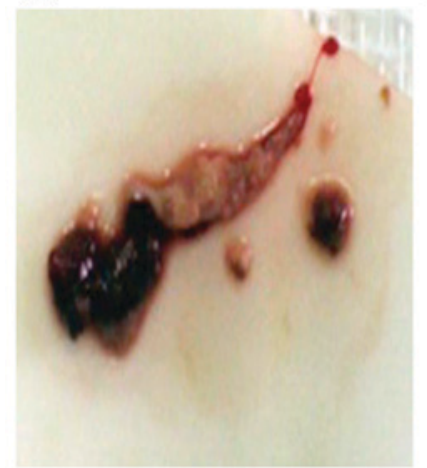

C

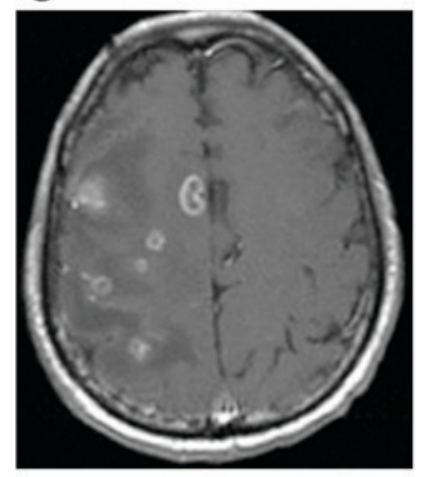

B

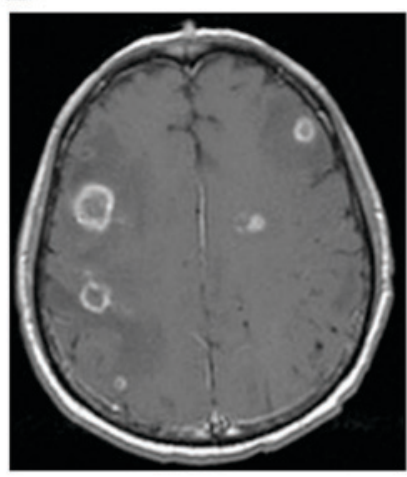

D

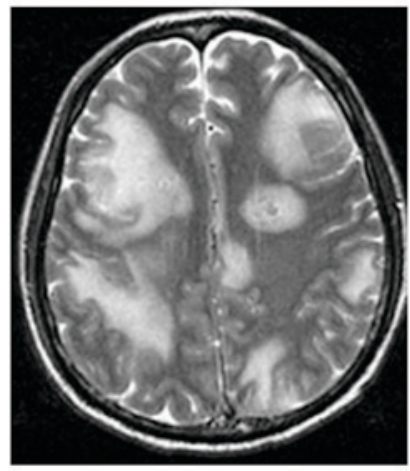

Figure 4. The MRI and biopsy material of a 20 -year-old male with acute lymphoblastic leukemia. (A) Stereotactic biopsy drew 'yellow red' cystic fluid from the right frontal lobe lesion with damaged and necrotic tissue appearing as dark 'broken cotton'. (B) T1-weighted MRI with gadopentetic acid shows multiple small capsule enhancing lesions on the bilateral cerebral hemisphere cortex and subcortical. (C) T1-weighted MRI with gadopentetic acid shows multiple small capsule enhancing lesions on the right cerebral hemisphere. (D) T2-weighted MRI shows long T2 signal lesions on the bilateral cerebral hemisphere with unclear border and apparent perilesional edema. MRI, magnetic resonance imaging.

intense anti-leukemia chemotherapy, or anti-GVHD immunosuppressive therapy following allo-HSCT.

Among the eight patients with CNS leukemia, seven were pathologically confirmed by stereotactic biopsy. The pathology of CNS leukemia was variable, which led to complex and changeable presentations during MRI. The pathological presentations of five patients were of tumor cells with nodular infiltration in the brain parenchyma. MRI scans exhibited a scattered, round and solid nodule of lesions in the brain parenchyma, with a slightly long or equal T1 and long T2 signal. Perilesional edema was marked, as was an enhanced mass-effect. One patient's pathology results indicated that tumor cells had infiltrated the meninges and meningeal vessels. The 'lace' strengthening of the meninges and the thickening of local meninges were observed using MRI. Tumor cells infiltrated and blocked vessels of the parenchyma in one patient and this patient's MRI scans revealed a partial small infarct, infarction, hemorrhagic infarction, or venous stasis of brain edema or cerebral hemorrhage.

When patients with CNS infections or neurodegenerative disorders demonstrated nodular infiltration lesions, these lesions tended to be confused with CNS leukemia on MRI scans. MRI of case 13 exhibited enhanced lesions in the left parietal and occipital lobe with surrounding edema, which 
was misdiagnosed as CNS leukemia. MRI of case 2 revealed a cystic lesion in the right temporal lobe and cerebellum, as well as marked edema, which were misdiagnosed as a CNS infection.

MRS allows for the noninvasive acquisition of biochemical information from biological tissues. Within a defined volume of interest, signals are detected from chemical nuclei, with protons (hydrogen ions) being most frequently used (35). During the present study, in patients with CNS leukemia MRS consistently demonstrated an increase in Cho, and a decrease in or absence of NAA. The $\mathrm{Cr}$ peak was consistent with previous data from the literature $(36,37)$. This indicates the 'exogenous' features of the tumor, which possibly aid the differentiation of CNS leukemia from other lesions.

Due to the diversity in pathological changes and imaging findings within CNS leukemia, it is challenging to identify the precise nature of CNS lesions in patients with leukemia, which is used to determine the type of treatment $(38,39)$. Various studies have demonstrated that the misdiagnosis rate of CNS leukemia may be $\leq 75 \%$, with misdiagnoses including intracranial hemorrhage, cerebral infarction, meningitis, infection, demyelinating multiple sclerosis, spinal cord compression syndrome and Guillain-Barre syndrome $(38,39)$. It is possible to obtain pathological confirmation safely through stereotactic biopsy with minimal trauma $(21,22)$. Clinical misdiagnosis and administering experimental treatments delays the correct treatment, which is an important factor for tumor recurrence (13). Therefore, the medical history, hematology and bone marrow test, CSF and biochemical examination, in combination with the pathology of CNS lesions following stereotactic biopsy, may improve the rate of correct diagnosis (23), avoiding unnecessary treatment and associated morbidity. At the same time, attention must be given to the function of blood coagulation prior to biopsy as numerous patients with leukemia have blood coagulation dysfunctions (32). In the present study, 12 patients with leukemia with CNS lesions underwent stereotactic biopsy without surgical complications for the pathological confirmation of diagnosis. Stereotactic biopsy has the advantages of convenience, as well as minimal invasion $(21,22)$.

The incidence of CNS lesions in leukemia has increased due to advances in treatment and prolonged survival time $(6,7)$. CNS leukemia typically presents as a scattered, round solid nodule of lesions in the brain parenchyma that reflects tumor cell nodular infiltration. These lesions typically exhibit slightly long or equal T1 and long T2 signal, with marked perilesional edema, mass-effect and contrast enhancement $(13,20)$. Differential diagnoses of CNS leukemia on MRI scans include CNS infection in immunocompromised patients and neurodegenerative disorders caused by anti-leukemia treatment $(14,20)$.

The novel imaging technique ${ }^{1} \mathrm{H}-\mathrm{MRS}$ is important in the diagnosis of CNS leukemia, and in differentiating it from other brain lesions in patients with leukemia. This is particularly vital when the characteristic imaging findings that usually appear on traditional images are absent (37-41).

To conclude, the present study demonstrated that the clinical diagnosis was not consistent with the pathological diagnosis in $2 / 14$ patients. Numerous CNS lesions in patients with leukemia are potentially curable; therefore, correct diagnosis is crucial.
Pathological confirmation remains the gold standard for diagnosing the nature of CNS lesions. In addition, the present study demonstrated that stereotactic biopsy is useful in diagnosing and differentiating CNS lesions in patients with leukemia. This technique may aid early recognition of the nature of CNS lesions and potentially allow for timely therapeutic intervention.

\section{Acknowledgements}

The present study was supported by the National Natural Science Foundation of China General Program (grant no. 81272842) and the Beijing Natural Science Foundation (grant no. 7172071).

\section{References}

1. Parkin DM, Ferlay J, Curado MP, Bray F, Edwards B, Shin HR and Forman D: Fifty years of cancer incidence: C15 I-IX. Int J Cancer 127: 2918-2927, 2010.

2. Lei T, Mao WM, Yang HJ, Chen XZ, Lei TH, Wang XH, Ying Q, Chen WQ and Zhang SW: Study on cancer incidence through the cancer registry program in 11 cities and counties, China. Zhonghua Liu Xing Bing Xue Za Zhi 30: 1165-1170, 2009 (In Chinese)

3. Chen WQ, Shan BE, Zheng RS, Lin GZ, Chen JZ, Chen JG and HE YT: Analysis of incidence and mortality of leukemia in registration areas of China from 2003 to 2007. Tumor 32: 251-255, 2012.

4. Rodriguez-Abreu D, Bordoni A and Zucca E: Epidemiology of hematological malignancies. Ann Oncol 18: 3-8, 2007.

5. Allemani C, Weir HK, Carreira H, Harewood R, Spika D, Wang XS, Bannon F, Ahn JV, Johnson CJ, Bonaventure A, et al: Global surveillance of cancer survival 1995-2009: Analysis of individual data for $25,676,887$ patients from 279 population-based registries in 67 countries (CONCORD-2). Lancet 385: 977-1010, 2015

6. Evans AE, Gilbert ES and Zandstra R: The increasing incidence of central nervous leukemia in children (Children's Cancer Study Group A). Cancer 26: 404-409, 1970.

7. Niemeyer CM, Hitchcock-Bryan S and Sallan SE: Comparative analysis of treatment programs for childhood acute lymphoblastic leukemia. Semin Oncol 12: 122-130, 1985.

8. Walker RW: Neurologic complications of leukemia. Neurol Clin 9: 989-999, 1991

9. Feinberg WM and Swenson MR: Cerebrovascular complications of L-asparaginase therapy. Neurol 38: 127-133, 1988.

10. Flament-Durand J, Ketelbant-Balasse P, Maurus R, Regnier R and Spehl M: Intracerebral calcifications appearing during the course of acute lymphocytic leukemia treated with methotrexate and X rays. Cancer 35: 319-325, 1975.

11. Rubinstein LJ, Herman MM, Long TF and Wilbur JR: Disseminated necrotizing leukoencephalopathy: A complication of treated central nervous system leukemia and lymphoma. Cancer 35: 291-305, 1975.

12. Biti GP, Magrini SM, Villari N, Caramella D, Guazzelli G, Rosi A and Lippi A: Brain damage after treatment for acute lymphoblastic leukemia. A report on 34 patients with special regard to MRI findings. Acta Oncol 28: 253-256, 1989.

13. Chen CY, Zimmerman RA, Faro S, Bilaniuk LT, Chou TY and Molloy PT: Childhood leukemia: Central nervous system abnormalities during and after treatment. AJNR Am J Neuroradiol 17: 295-310, 1996

14. Faraci M, Lanino E, Dini G, Fondelli MP, Morreale G, Dallorso S, Manzitti C, Calevo MG, Gaggero R, Castagnola E and Haupt R: Severe neurologic complications after hematopoietic stem cell transplantation in children. Neurology 59: 1895-1904, 2002.

15. Pui $\mathrm{CH}$ and Thiel E: Central nervous system disease in hematologic malignancies: Historical perspective and practical applications. Semin Oncol 36 (4 Suppl 2): S2-S16, 2009.

16. Phillips ME, Ryals TJ, Kambhu SA and Yuh WT: Neoplastic vs inflammatory meningeal enhancement with Gd-DTPA. J Comput Assist Tomogr 14: 536-541, 1990. 
17. Ball WS Jr, Prenger EC and Ballard ET: Neurotoxicity of radio/chemotherapy in children: Pathologic and MR correlation. AJNR Am J Neuroradiol 13: 761-776, 1992.

18. Asato R, Akiyama Y, Ito M, Kubota M, Okumura R, Miki Y, Konishi J and Mikawa H: Nuclear magnetic resonance abnormalities of the cerebral white matter in children with acute lymphoblastic leukemia and malignant lymphoma during and after central nervous system prophylactic treatment with intrathecal methotrexate. Cancer 70: 1997-2004, 1992.

19. Tsuruda JS, Kortman KE, Bradley WG, Wheeler DC, Van Dalsem W and Bradley TP: Radiation effects on cerebral white matter: MR evaluation. AJR Am J Roentgenol 149: 165-171, 1987.

20. Packer RJ, Zimmerman RA and Bilaniuk LT: Magnetic resonance imaging in the evaluation of treatment-related central nervous system damage. Cancer 58: 635-640, 1986.

21. Ligima K, Hirato M, Miyagishima T, Horiguchi K, Sugawara K Hirato J, Yokoo $\mathrm{H}$ and Yoshimoto Y: Microrecording and image-guided stereotactic biopsy of deep-seated brain tumors. J Neurosurg 123: 978-988, 2015.

22. Malikova H, Liscak R, Latnerova I, Guseynova K, Syrucek M and Pytlik R: Complications of MRI-guided stereotactic biopsy of brain lymphoma. Neuro Endocrinol Lett 35: 613-618, 2014.

23. Göçmen S, Kutlay M,Erikçi A, Atabey C, Sayan O and Haholu A Central nervous system involvement of T-cell prolymphocytic leukemia diagnosed with stereotactic brain biopsy: Case report. Turk J Haematol 31: 75-78, 2014.

24. Thomas X, Boiron JM, Huguet F, Dombret H, Bradstock K Vey N, Kovacsovics T, Delannoy A, Fegueux N, Fenaux P, et al: Outcome of treatment in adults with acute lymphoblastic leukemia: Analysis of LALA-94 trial. J Clin Oncol 22: 4075-4086, 2004.

25. Kantarjian HM, O'Brien S, Smith TL, Cortes J, Giles FJ, Beran M, Pierce S, Huh Y, Andreeff M, Koller C, et al: Results of treatment with hyper-CVAD, a dose-intensive regimen, in adult acute lymphocytic leukemia. J Clin Oncol 18: 547-561, 2000

26. Thomas $X$ and Le QH: Central nervous system involvement in adult acute lymphoblastic leukemia. Hematology 13: 293-302, 2008.

27. Sung SH and Jang IS: Isolated central nervous system relapse of acute lymphoblastic leukemia. Brain Tumor Res Treat 2: 114-118, 2014.

28. Barredo $\mathrm{J}$ and Ritchey AK: Controversies in the management of central nervous system leukemia. Pediatr Hematol Oncol 27: 329-332, 2010.

29. Robertson KA: Pediatric bone marrow transplantation. Curr Opin Pediatr 5: 103-109, 1993.

30. Pui $\mathrm{CH}$ and Howard SC: Current management and challenges of malignant disease in the CNS in paediatric leukemia. Lancet Oncol 9: 257-268, 2008
31. Valk PE and Dillon WP: Radiation injury of the brain. AJNR Am J Neuroradiol 12: 45-62, 1991.

32. Priest JR, Ramsay NK, Steinherz PG, Tubergen DG, Cairo MS, Sitarz AL, Bishop AJ, White L, Trigg ME, Levitt CJ, et al: A syndrome of thrombosis and hemorrhage complicating L-asparaginase therapy for childhood acute lymphoblastic leukemia. J Pediatr 100: 984-989, 1982.

33. Price RA and Jamieson PA: The central nervous system in childhood leukemia. II. Subacute leukoencephalopathy. Cancer 35: 306-318, 1975.

34. Törnebohm E, Lockner D and Paul C: A retrospective analysis of bleeding complications in 438 patients with acute leukemia during the years 1972-1991. Eur J Haematol 50: 160-167, 1993.

35. Mansour A, Qandeel M, Abdel-Razeq H and Abu Ali HA: MR imaging features of intracranial primary CNS lymphoma in immune competent patients. Cancer Imaging 14: 22, 2014

36. Chan YL, Roebuck DJ, Yuen MP, Yeung KW, Lau KY, Li CK and Chik KW: Long-term cerebral metabolite changes on proton magnetic resonance spectroscopy in patients cured of acute lymphoblastic leukemia with previous intrathecal methotrexate and cranial irradiation prophylaxis. Int J Radiat Oncol Biol Phys 50: 759-763, 2001.

37. Ficek K, Blamek S, Syguła D, Miszczyk L, Sońta-Jakimczyk D and Tarnawski R: Evaluation of the late effects of CNS prophylactic treatment in childhood acute lymphoblastic leukemia (ALL) using magnetic resonance spectroscopy. Acta Neurochir Suppl 106: 195-197, 2010.

38. Kumar R, Nijalingappa S, Grainger J and Ismayl O: Acute disseminated encephalomyelitis mimicking late CNS relapse of acute lymphoblastic leukaemia: Case report. J Med Case Rep 1: 4, 2007

39. Ferrés GM, Bidart HT and Zubieta AM: Important of images and etiological diagnosis of central nervous system involvement in immunocompromised patients. Rev Chilena Infectol 27: 541-543, 2010 (In Spanish).

40. Brandt MD, Brandt K, Werner A, Schönfeld R, Loewenbrück K, Donix M, Schaich M, Bornhäuser M, von Kummer R, Leplow B and Storch A: Preventive brain radio-chemotherapy alters plasticity associated metabolite profile in the hippocampus but seems to not affect spatial memory in young leukemia patients. Brain Behav 5: e00368, 2015.

41. Chan YL, Roebuck DJ, Yuen MP, Yeung KW, Lau KY, Li CK and Chik KW: Long-term cerebral metabolite changes on proton magnetic resonance spectroscopy in patients cured of acute lymphoblastic leukemia with previous intrathecal methotrexate and cranial irradiation prophylaxis. Int J Radiat Oncol Biol Phys 50: 759-763, 2001 